\title{
Successful pregnancy and delivery in patient with double uterus and in vitro fertilization
}

\begin{abstract}
The presence of uterus didelphys is a rare pathology that does not significantly affect the chances of achieving pregnancy in the absence of other factors of infertility. We present a case of a patient with a proven abnormality - the presence of uterus didelphys - in which an IVF-ET procedure was performed due to male infertility factor. After embryo transfer of 1 embryo in each uterus, a singleton pregnancy was realized in the one uterine body. After a normal pregnancy, the patient was delivered near the due date by cesarean section without complications for the mother or fetus. This case proved the hypothesis that patients with a double uterus have a good prognosis for pregnancy. In this case, male infertility was the leading cause of infertility and after a successful in vitro procedure, a pregnancy was achieved, which ended with a successful term delivery.
\end{abstract}

Keywords: uterus didelphys, in vitro fertilization
Volume 10 Issue 4 - 202 I

\author{
Gergana Ingilizova,' Sergei Slavov, ${ }^{2,3}$ Galina \\ Yaneva ${ }^{4}$ \\ 'Vita" Multidisciplinary Hospital for Active Treatment, Bulgaria \\ 2University Hospital of Obstetrics and Gynecology "Maichin \\ Dom", Bulgaria \\ ${ }^{3}$ Department of Obstetrics and Gynecology, Medical University, \\ Bulgaria \\ ${ }^{4}$ Department of Biology, Medical University of Varna, Bulgaria
}

Correspondence: Gergana Ingilizova, “Vita” Multidisciplinary Hospital for Active Treatment, Sofia, Bulgaria,

Tel +359887204480, ORCID 0000-0002-0718-458I,

Email ingilizova@mail.bg

Received: June 15, 2021 | Published: July 21, 202

\section{Introduction}

Uterus didelphys accounts for around $8-10 \%$ of all Müllerian anomalies. In this type of anomaly there are two uterine horns with separate noncommunicating uterine cavities, each associated with its own uterine cervix and fallopian tube. Uterus didelphys occurs in approximately 1 in 3000 women. ${ }^{1}$ Compared with other uterine anomalies uterus didelphys has a relatively good prognosis for achieving pregnancy, but still there is an increased risk for miscarriage and preterm delivery. ${ }^{2,3}$ Congenital uterine anomalies had no effect on the rate of clinical pregnancy when performing assisted reproduction. However, it is proven, that major uterine anomalies lead to a significantly worse obstetric outcomes. ${ }^{4}$ We here report a case of woman with double uterus and successful pregnancy after In vitro fertilization.

\section{Case report}

The case conserns a 37-year-old patient with primary infertility because of male factor, who participated in a fertility treatment programme in "Vita" Multidisciplinary Hospital for Active Treatment, Sofia, Bulgaria in June 2020. All necessary tests have been performed before the In Vitro procedure. Sperm analysis showed oligoasthenozoospermia, according to World health organization(WHO) criteria. Hormonal tests showed a normal ovarian reserve - anti Mullerian hormone $1.7 \mathrm{ng} / \mathrm{ml}$. The patient reported regular menstrual cycle. Hashimoto's thyroiditis and anemia were concomitant diseases. During the performed diagnostic procedures the presence of an anomaly of the Muellerian duct - uterus didelphys was established. The diagnosis was made by physical examination, which revealed the presence of two cervixes and subsequent hysteroscopy, where two independent uterine bodies were found. The diagnose was also confirmed by 3D ultrasound (Figure 1). The patient was offered an in vitro procedure and, after preliminary preparation, controlled ovarian hyperstimulation was performed. A protocol with a gonadotropin antagonist (Cetrorelix) and recombinant folliclestimulating hormone (Gonal-F, $1200 \mathrm{IU}$ ) was used. Oocyte retrieval was carried out on the 12th day of stimulation. Seven follicles were aspirated and 3 MII oocytes were collected. Intracytoplasmic sperm injection (ICSI) was performed and two of the oocytes were normally fertilized. One G1 (excellent quality) and one G2 (very good quality) embryos were achieved. On day 3 , embryo transfer of one embryo was performed in each of the uterine bodies. Luteal maintenance with progesterone and estradiol was prescribed. After 12 days, the patient showed a positive HCG result. Ultrasound examination showed pregnancy into the one uterus. After a normal pregnancy, the patient was delivered at 38 weeks of gestation by cesarean section. The presence of two uteruses with pregnancy in one was confirmed intraoperatively. The fetus was in a caphalic presentation and weighed 3420 g. The technique used during cesarean section was a lower transverse incision of the uterus. Two myomas were found in the nonpregnant uterus, which were removed. The postpartum and neonatal periods passed without complications.

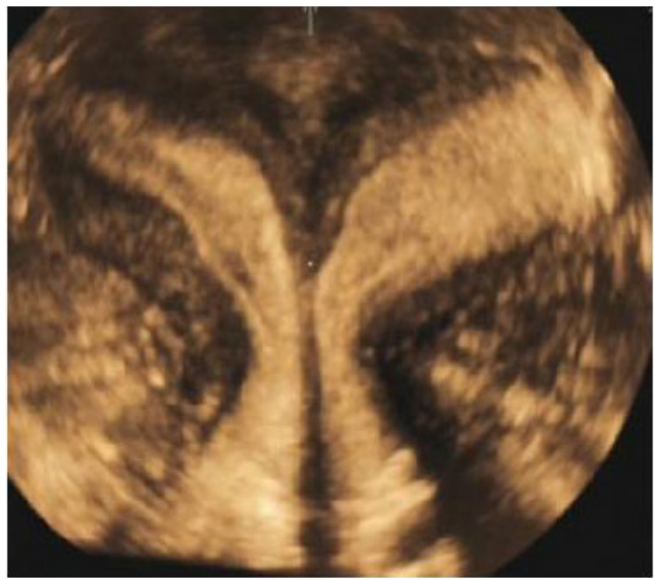

Figure I Ultrasound image of the uterus.

\section{Discussion}

Uterus didelphys is rarely the sole cause of infertility. In our case, additional factors such as male infertility played a major role in the 
decision to perform in vitro fertilization, but also increased maternal age and related to it decreased ovarian reserve may play role. ${ }^{6,7}$ It has been shown that the fertility of women with untreated uterus dydelphys is better than those with other Mullerian duct abnormalities, but it is still lower than women with normal uterine anatomy. There is also an increased risk of miscarriage, fetal growth retardation and premature delivery. ${ }^{8}$ Other authors didn't find significant difference in the average number of oocytes retrieved, the fertilization and embryo cleavage rates or the mean number of embryos transferred when comparing the different types of malformation. ${ }^{9}$ They found that mean number of oocytes retrieved was 9.3 and the mean number of embryos transferred per cycle was 2.8 , which is a very similar result to that in our case.

Split embryo transfer into each uterine cavity of uterus didelphys with successful pregnancy and delivery has been reported by other authors. ${ }^{10}$ The purpose of embryo transfer in both uterine cavities is to increase the rate of conception, but the desired outcome is to achieve a singleton pregnancy, as pregnancy with twins may be associated with additional complications. ${ }^{11}$ The rate of delivery with cesarean section in cases with uterus didelphys is higher than in general population. ${ }^{8}$ Perhaps the higher incidence of cesarean section in these cases is due to the fear of a worse obstetric outcome, especially in women with a previous cesarean section and the risk of uterine rupture. ${ }^{12}$ This is part of risk management in such patients and related to the prevention of complications. ${ }^{13-16}$

\section{Conclusion}

Uterus didelphys is a rare pathology which is associated with good pregnancy rates when IVF-ET is performed compared with those in the general infertile population. Pregnancy in these patients should be closely monitored given the higher risk of preterm delivery and the higher rate of cesarean delivery. It is recommended that embryo transfer of up to 2 embryos be performed in patients with a good prognosis. ${ }^{17}$

\section{Acknowledgments}

None.

\section{Conflicts of interest}

The author declares no conflict of interest

\section{Funding}

None.

\section{References}

1. Grimbizis GF, Camus M, Tarlatzis BC, et al. Clinical implications of uterine malformations and hysteroscopic treatment results. Hum Reprod Update. 2001;7(2):161-74.
2. Felker EA. Uterus Didelphys and Pregnancy. Journal of Diagnostic Medical Sonography. 2004; 20(2):131-133.

3. Taylor E, Gomel V. The uterus and fertility. Fertility and Sterility. 2008;89(1):1-16

4. Prior M, Richardson A, Asif S et al. Outcome of assisted reproduction in women with congenital uterine anomalies: a prospective observational study. Ultrasound Obstet Gynecol. 2018;51(1):110-117.

5. Veeck LL. The morphological assessment of human oocytes and early concepti, Handbook of the Laboratory Diagnosis and Treatment of Infertility (eds B. A. Keel, B. W. Webster), Boca Raton, FL, CRC Press. 1990:353-369.

6. Yunakova M, Surcheva H, Andreeva P, et al. Comparison of prognostic value of markers of ovarian reserve. Akusherstvo I ginekologia. 2017;56(6):3-9.

7. Yunakova M, Gogova G, Antonova I, et al. Age as a predictive marker of assisted reproductive technologies outcome. Akusherstvo I ginekologia. 2017;56(6):17-21.

8. Rezai S, Bisram P, Lora Alcantara, et al. Didelphys Uterus: A Case Report and Review of the Literature. Case Rep Obstet Gynecol. 2015;2015:865821.

9. Marcus S, A1-Shawaf T, Brinsden P. The obstetric outcome of in vitro fertilization and embryo transfer in women with congenital uterine malformation American Journal of Obstetrics and Gynecology. 1996;175(1):85-89.

10. Al-Hussaini TK. Two successful pregnancies using split embryo transfer in a woman with uterus didelphys: A case report. Middle East Fertility Society Journal. 2017;22(1):70-72.

11. Yang MJ, Tseng JY, et al. Delivery of double singleton pregnancies in a woman with a double uterus, double cervix, and complete septate vagina J Chin Med Assoc. 2015;78(12):746-748.

12. Kostov I, Garnizov T. Uterine rupture at birth after previous sectio delivery with minimal dosing of Oxytocin - case report. Akusherstvo I ginekologia. 2017;56(8):28-30.

13. Kostov I, Ivanov St. Prevention and prophylaxis in obstetrics and gynecology. Akusherstvo i Ginekologia. 2018;57(2):57-58.

14. Kostov I, Ivanov St. Recommendation for intrapartum management of intraamniotic infections. Akusherstvo i Ginekologiia. 2018;57(2):50-56.

15. Kostov I, Yunakova M, Georgiev B, et al. Analysis of the quality of medical services in obstetrics and gynecology practice in Bulgaria - a study in organizational, clinical and deontological aspects. Akusherstvo I ginekologia. 2017;56(6):9-16.

16. Ignatov P, Atanasov B, Kostov I et al. A comparative study of the prognostic $\mathrm{pH}$ values of the fetus in utero, generated by the "quantitative cardiotocography" computer method, and the actual $\mathrm{pH}$ values measured immediately after delivery. Akusherstvo $i$ Ginekologiia. 2010;49(4):3-11. 